\title{
Projective Limit of Haar Measures on $O(n)$
}

\author{
By \\ Yasuo YAMASAKI
}

\section{Introduction}

In this paper, we shall show that the Gaussian measure [1] on $R^{\infty \infty}$ is obtained as the projective limit of Haar measures on $O(n)$. This is a natural extension of the fact [2] [3]: the Gaussian measure on $R^{\infty}$ is obtained as the projective limit of the uniform measures on the $n$ dimensional spheres.

D. Shale [4] considered the family of Haar measures on $O(n)$ to construct a finitely additive measure on $O(\infty)$. But he did not treat the projective limit. Also a report by $H$. Shimomura $[5]$ is useful for the information on this topic.

\section{§1. Orthogonal Group $\boldsymbol{O}(\boldsymbol{n})$}

The $n$-dimensional orthogonal group $O(n)$ is the group of all orthogonal transformations of $R^{n}$. If we fix a C.O.N.S. (= complete orthonormal system) of $R^{n}$, it is identified with the group of all matrices $\left(u_{i j}\right)$ which satisfy the orthogonality relations:

$$
\sum_{k=1}^{n} u_{i k} u_{j k}=\delta_{i j} \quad(1 \leqq i, j \leqq n)
$$

Because of (1.1), only $n(n-1) / 2$ matrix elements are independent, and the other $n(n+1) / 2$ matrix elements can be considered as functions of the formers.

Usually, $n(n-1) / 2$ Euler angles are used as independent variables of 
$O(n)$, but in this paper, for the convenience of later analysis, we shall use another system of independent variables which we shall explain in the last part of this section.

Let $S_{n-1}$ be the unit sphere of $R^{n}$;

$$
S_{n-1}=\left\{\left(x_{1}, x_{2}, \ldots, x_{n}\right) ; x_{1}^{2}+x_{2}^{2}+\cdots+x_{n}^{2}=1\right\} .
$$

The group $O(n)$ can be regarded as a transformation group of $S_{n-1}$. The group of all orthogonal transformations which keep the vector $(0,0, \ldots$, $0,1)$ invariant, is isomorphic with $O(n-1)$, so we identify them. For any $U, V \in O(n)$, we have $U V^{-1} \in O(n-1)$, if and only if the last row vector of $U$ is equal with that of $V$, namely

$$
u_{n j}=v_{n j} \quad(1 \leqq j \leqq n) .
$$

Therefore, the coset space $O(n-1) \backslash O(n)$ is identified with $S_{n-1}$. Suppose that a mapping $S_{n-1} \ni x \rightarrow U_{x} \in O(n)$ is given such that the last row vector of $U_{x}$ is just $x$. In other words, each $U_{x}$ is a representative of the coset which corresponds to $x$. Then, any $U \in O(n)$ is written uniquely in the form:

$$
U=\left(\begin{array}{cc}
U_{1} & 0 \\
0 & 1
\end{array}\right) U_{x} \quad U_{1} \in O(n-1), x \in S_{n-1} \text {. }
$$

For $V \in O(n)$, the last row vector of $U_{x} V$ is $x V$. So, if $U$ is represented as (1.4), we have

$$
\begin{aligned}
U V & =\left(\begin{array}{ll}
U_{1} & 0 \\
0 & 1
\end{array}\right) U_{x} V=\left(\begin{array}{cc}
U_{1} & 0 \\
0 & 1
\end{array}\right)\left(\begin{array}{ll}
W & 0 \\
0 & 1
\end{array}\right) U_{x V} \\
& =\left(\begin{array}{ll}
U_{1} W & 0 \\
0 & 1
\end{array}\right) U_{x V} \quad \text { for some } W \in O(n-1) .
\end{aligned}
$$

Therefore, any multiplication from right on $O(n)$ induces (1) a multiplication from right on $O(n-1)$, and (2) an orthogonal transformation on $S_{n-1}$.

Consider the uniform measure on $S_{n-1}$ and the Haar measure on $O(n-1)$. From the above discussion, we see that their product measure 
is just the Haar measure on $O(n)$, if we identify $U \in O(n)$ with $\left(U_{1}, x\right)$ $\in O(n-1) \times S_{n-1}$. Here we assume that the mapping $x \rightarrow U_{x}$ is measurable, but this assumption is satisfied if $U_{x}$ is defined and continuous except on some closed null set of $S_{n-1}$.

Now, we shall define concretely the mapping $x \rightarrow U_{x}$. Let $\left\{e_{1}, e_{2}, \ldots\right.$, $\left.e_{n}\right\}$ be the C.O.N.S. of $R^{n}$. If $x_{n}=\left\langle x, e_{n}>\neq 0\right.$, the vectors $x, e_{1}$, $e_{2}, \ldots, e_{n-1}$ are linearly independent. Then, we adopt the Schmidt's orthonormalization of them as row vectors of $U_{x}$.

Explicitly writing, the matrix elements of $U_{x}$ is as follows;

$$
\left\{\begin{array}{l}
U_{x}=\left(u_{i j}\right) \\
u_{n j}=x_{j} \\
u_{i j}=0 \quad \text { if } j<i \leqq n-1 \\
u_{i i}=\sqrt{x_{i+1}^{2}+\cdots+x_{n}^{2}} / \sqrt{x_{i}^{2}+\cdots+x_{n}^{2}} \quad \text { if } \quad i \leqq n-1 \\
u_{i j}=-x_{i} x_{j} / \sqrt{x_{i}^{2}+\cdots+x_{n}^{2}} \sqrt{x_{i+1}^{2}+\cdots+x_{n}^{2}} \quad \text { if } i<j .
\end{array}\right.
$$

Hereafter, let the mapping $x \rightarrow U_{x}$ be always the above one.

Since $O(n) \simeq O(n-1) \times S_{n-1}$, repeating the similar procedure, we have $O(n) \simeq S_{1} \times S_{2} \times \cdots \times S_{n-1}$. Then, the Haar measure on $O(n)$ is the product measure of uniform measures on $S_{k}(1 \leqq k \leqq n-1)$. More exactly speaking, we can formulate as follows. Let $\varphi_{n}$ be the mapping $O(n) \rightarrow O(n-1)$ such that

$$
\varphi_{n}(U)=U_{1} \quad \text { where } U=\left(\begin{array}{cc}
U_{1} & 0 \\
0 & 1
\end{array}\right) U_{x}
$$

Then the mapping $\varphi_{k+1} \circ \varphi_{k+2} \circ \ldots \circ \varphi_{n}$ maps $O(n)$ to $O(k)$. Denote the matrix elements of the image matrix as $u_{i j}^{(k)}(1 \leqq i, j \leqq k)$. They are functions on $O(n)$.

If we adopt $u_{k j}^{(k)}(1 \leqq j \leqq k-1,2 \leqq k \leqq n)$ as $n(n-1) / 2$ independent variables of $O(n)$, the Haar measure $\mu_{n}$ on $O(n)$ is represented as follows (except normalization constant): 


$$
d \mu_{n}=\prod_{k=2}^{n}\left[\left\{1-\sum_{j=1}^{k-1} u_{k j}^{(k) 2}\right\}^{-\frac{1}{2}} \prod_{j=1}^{k-1} d u_{k j}^{(k)}\right]
$$

the content of [] being the uniform measure on $S_{k-1}$.

\section{§2. Projective Limit}

Since the Haar measures $\mu_{n}$ satisfy

$$
\mu_{n-1}(A)=\mu_{n}\left(\varphi_{n}^{-1}(A)\right) \quad \text { for a Borel subset } A \text { of } O(n-1) \text {, }
$$

according to a theorem due to Bochner, we can construct the projective limit probability space $(\Omega, \mathcal{B}, \mu)$. It satisfies the following properties:

P1) $\Omega \subset \prod_{n=1}^{\infty} O(n)$

P2) $f_{n-1}=\varphi_{n} \circ f_{n}$. Here, $f_{n}$ is the restriction of $\pi_{n}$ on $\Omega$, where $\pi_{n}$ is the projection from $\prod_{n=1}^{\infty} O(n)$ onto $O(n)$.

P3) $B$ is generated by $\bigcup_{n=1}^{\infty} f_{n}^{-1}\left(B_{n}\right)$, where $B_{n}$ is the whole of Borel subsets of $O(n)$.

P4) $\mu\left(f_{n}^{-1}(A)\right)=\mu_{n}(A) \quad$ for $A \in B_{n}$.

The matrix elements $u_{i j}^{(k)}(1 \leqq i, j \leqq k)$ can be regarded as functions on $\Omega$, as well as on $O(n)$ for $n \geqq k$. Then, for any $\omega \in \Omega$,

$$
f_{k}(\omega)=\left(u_{i j}^{(k)}(\omega)\right) \in O(k) .
$$

$u_{i j}^{(k)}(\omega)$ is a measurable function on $\Omega$ because of P3).

\section{Lemma 1 .}

$$
\int_{\Omega} u_{i j}^{(n)}(\omega) d \mu=0
$$

$$
\int_{\Omega} u_{i j}^{(n)}(\omega) u_{p q}^{(m)}(\omega) d \mu=\delta_{i p} \delta_{j q} \frac{1}{m} \frac{\Gamma\left(\frac{n-j+1}{2}\right)}{\Gamma\left(\frac{n-j+2}{2}\right)} \frac{\Gamma\left(\frac{m-j+2}{2}\right)}{\Gamma\left(\frac{m-j+1}{2}\right)}
$$

for $m \leqq n$, where $\Gamma$ is Gamma function. 
Proof. From P4), we have

$$
\int_{\Omega} u_{i j}^{(n)}(\omega) d \mu=\int_{O(n)} u_{i j}^{(n)} d \mu_{n}=0
$$

Similarly, using 2) also, we see that

$$
\int_{\Omega} u_{i j}^{(n)}(\omega) u_{p q}^{(m)}(\omega) d \mu=\int_{O(n)} u_{i j}^{(n)} u_{p q}^{(m)} d \mu_{n}
$$

Substituting (1.8),

$$
=\int_{O(m)} I_{i j} u_{p q}^{(m)} d \mu_{m}
$$

where $I_{i j}=$ integration of $u_{i j}^{(n)}$ by $\prod_{k=m}^{n-1} \int_{O(k) \backslash O(k+1)} d m_{k}, m_{k}$ being the uniform measure on $S_{k}$.

Now, we shall calculate

$$
I^{(n-1)}=\int_{O(n-1) \backslash O(n)} U^{(n)} d m_{n-1}
$$

Since $U^{(n)}=\left[\begin{array}{ll}U^{(n-1)} & 0 \\ 0 & 1\end{array}\right] U_{x}$, we have

$$
I^{(n-1)}=\int_{S_{n-1}}\left[\begin{array}{ll}
U^{(n-1)} & 0 \\
0 & 1
\end{array}\right] U_{x} d m_{n-1}=\left[\begin{array}{ll}
U^{(n-1)} & 0 \\
0 & 1
\end{array}\right] \int_{S_{n-1}} U_{x} d m_{n-1} .
$$

Using (1.6), we have

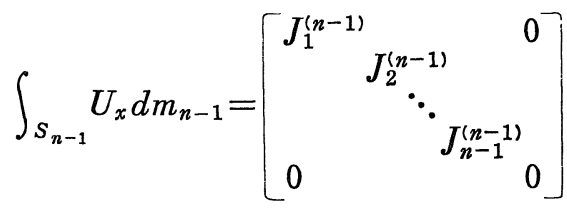

where $J_{i}^{(n-1)}=\int_{S_{n-1}} \frac{r_{n-i}}{r_{n-i+1}} d m_{n-1}=\int_{0}^{\pi} \sin ^{n-i} \theta d \theta / \int_{0}^{\pi} \sin ^{n-i-1} \theta d \theta$. Thus we have

$$
I_{i j}=u_{i j}^{(m)} \prod_{k=m}^{n-1} J_{j}^{(k)}=u_{i j}^{(m)} \int_{0}^{\pi} \sin ^{n-j} \theta d \theta / \int_{0}^{\pi} \sin ^{m-j} \theta d \theta
$$




$$
=u_{i j}^{(m)} \frac{\Gamma\left(\frac{n-j+1}{2}\right)}{\Gamma\left(\frac{n-j+2}{2}\right)} \frac{\Gamma\left(\frac{m-j+2}{2}\right)}{\Gamma\left(\frac{m-j+1}{2}\right)} .
$$

On the other hand, since

$$
\int_{O(m)} u_{i j}^{(m)} u_{p q}^{(m)} d \mu_{m}=\frac{1}{m} \delta_{i p} \delta_{j q}
$$

we have (2.4) from (2.5) and (2.6).

Lemma 2. $\left\{\sqrt{n} u_{i j}^{(n)}(\omega) ; n \geqq \max (i, j)\right\}$ forms a Cauchy sequence in $L^{2}(\Omega, \mu)$. The speed of convergence is dependent on $j$, but uniform in $i$.

Proof. From (2.4), we have $\left\|\sqrt{n} u_{i j}^{(n)}\right\|=1$, and

$$
\left\langle\sqrt{n} u_{i j}^{(n)}, \sqrt{m} u_{i j}^{(m)}>=\sqrt{\frac{n}{m}} \frac{\Gamma\left(\frac{n-j+1}{2}\right)}{\Gamma\left(\frac{n-j+2}{2}\right)} \frac{\Gamma\left(\frac{m-j+2}{2}\right)}{\Gamma\left(\frac{m-j+1}{2}\right)} .\right.
$$

The latter tends to 1 as $n, m \rightarrow \infty$ because we have asymptotically

$$
\frac{\Gamma\left(t+\frac{1}{2}\right)}{\Gamma(t)} \sim \sqrt{t}
$$

From Lemma 2, $\sqrt{n} u_{i j}^{(n)}$ converges to a function $X_{i j}$ in $L^{2}(\Omega, \mu)$. Then, $X_{i j}$ is defined for almost all $\omega$, and some suitable subsequence of $\left\{\sqrt{n} u_{i j}^{(n)}(\omega)\right\}$ converges to $X_{i j}(\omega)$ almost everywhere. Evidently, we have

$$
\int_{\Omega} X_{i j}(\omega) d \mu=0 \quad \text { and } \quad \int_{\Omega} X_{i j}(\omega) X_{p q}(\omega) d \mu=\delta_{i p} \delta_{j q}
$$

\section{§3. Identification with the Gaussian Measure}

Proposition 1. For almost all $\omega, \omega^{\prime} \in \Omega$, the following (1) and (2) are equivalent. 
(1) $X_{i j}(\omega)=X_{i j}\left(\omega^{\prime}\right)$ for any $i, j$.

(2) $u_{i j}^{(n)}(\omega)=u_{i j}^{(n)}\left(\omega^{\prime}\right)$ for any $i, j, n$ where $n \geqq \max (i, j)$.

Proof. $\quad(2) \Rightarrow(1)$ is evident, because $X_{i j}(\omega)$ is the limit of some subsequence of $\left\{\sqrt{n} u_{i j}^{(n)}(\omega)\right\}$.

On the other hand, from the definition (1.6) of $U_{x}$, we see that for $U \in O(n)$, if $U=\left[\begin{array}{cc}U_{1} & 0 \\ 0 & 1\end{array}\right] U_{x}$, then the column vectors of $U_{1}$ is obtained by the Schmidt's orthonormalization of the projections to $R^{n-1}$ of the column vectors of $U$. Therefore, if $m \leqq n$, the column vectors of $U^{(m)}$ is the Schmidt's orthonormalization of the projections to $R^{m}$ of the column vectors of $U^{(n)}$, and in the limit of $n \rightarrow \infty$ (fixing $m$ ), $\sqrt{n}$ times of the matrix elements of $U^{(n)}$ tend to $X_{i j}$. So, $u_{i j}^{(m)}(1 \leqq i, j \leqq m)$ is obtained by the Schmidt's procedure from $X_{i j}$.

Q.E.D.

Consider the mapping $\psi: \omega \in \Omega \rightarrow\left(X_{i j}(\omega)\right) \in R^{\infty \infty}$, where $R^{\infty \infty}$ is the space of all double sequences. $\psi$ is one-to-one excpet on a suitable null set of $\Omega$, because $X_{i j}(\omega)=X_{i j}\left(\omega^{\prime}\right)$ for any $i, j$ implies $u_{i j}^{(n)}(\omega)=u_{i j}^{(n)}\left(\omega^{\prime}\right)$ for any $i, j, n$, therefore $f_{n}(\omega)=f_{n}\left(\omega^{\prime}\right)$ for any $n$, so that $\omega=\omega^{\prime}$.

Next, we shall discuss the measurability. From P3) of $\S 2$, the probability measure $\mu$ is defined on the smallest $\sigma$-ring $\mathcal{B}$ which makes all $u_{i j}^{(n)}(\omega)$ measurable. This is equivalent to say $B$ is the smallest $\sigma$-ring which makes $X_{i j}(\omega)$ measurable as seen from the proof of the proposition 1. Therefore the image $\psi(B)$ is the smallest $\sigma$-ring which makes all projections $\alpha=\left(\alpha_{i j}\right) \in R^{\infty} \rightarrow \alpha_{i j} \in R^{1}$ measurable. In other words, $\psi(\beta)$ is the smallest $\sigma$-ring which makes all Borel cylinder sets with the base in $R_{0}^{\infty \infty}$ measurable, where $R_{0}^{\infty \infty}$ is the space of all double sequences which vanish except for finite number of $(i, j)$.

Finally, we shall show that the measure $\mu$ on $\Omega$ is mapped to the Gaussian measure $g$ on $R^{\infty \infty}$.

For this purpose, we shall prove that

$$
\int_{\Omega} \exp \left[\sqrt{-1} \sum_{i, j} t_{i j} X_{i j}(\omega)\right] d \mu=\exp \left[-\frac{1}{2} \sum_{i, j} t_{i j}^{2}\right],
$$


where $t_{i j}$ are arbitrary real numbers and the summation is carried out for $i+j \leqq k$.

Since $\sqrt{n} u_{i j}^{(n)}(\omega)$ tends to $X_{i j}(\omega)$ in $L^{2}(\Omega, \mu)$, the left side of (3.1) is approximated by

$$
\int_{O(n)} \exp \left[\sqrt{-1} \sum_{i, j} t_{i j} \sqrt{n} u_{i j}^{(n)}\right] d \mu_{n}
$$

the error tending to 0 as $n \rightarrow \infty$.

The integral (3.2) is equal with

$$
\int_{O(n)} \exp \left[\sqrt{-1} \sum_{i, j} t_{i j} \sqrt{n} u_{n-i, j}^{(n)}\right] d \mu_{n}
$$

because $\mu_{n}$ is the Haar measure on $O(n)$.

Since the convergence $\sqrt{n} u_{i j}^{(n)} \rightarrow X_{i j}$ is uniform in $i, \sqrt{n} u_{n-i, j}^{(n)}$ in the integrand of (3.3) can be replaced by $\sqrt{n-i} u_{n-i, j}^{(n-i)}$ with good approximation, if $n$ is large enough and $i+j \leqq k$ for some fixed $k$. Namely, with small error the left side of (3.1) is approximated by

$$
\int_{O(n)} \exp \left[\sqrt{-1} \sum_{i, j} t_{i j} \sqrt{n-i} u_{n-i, j}^{(n-i)}\right] d \mu_{n}
$$

Substituting (1.8), this quantity is equal except the normalization constant of $\mu_{n}$ with

$$
\begin{gathered}
\int \exp \left[\sqrt{-1} \sum_{i, j} t_{i j} \sqrt{n-i} u_{n-i, j}^{(n-i)}\right] \prod_{i=1}^{k}\left[\left\{1-\sum_{j=1}^{k-i} u_{n-i, j}^{(n-i)^{2}}\right\}^{\frac{n-k-2}{2}} \prod_{j=1}^{k-i} d u_{n-i, j}^{(n-i)}\right] \\
=\int \exp \left[\sqrt{-1} \sum_{i, j} t_{i j} \lambda_{i j}\right] \prod_{i=1}^{k}\left[\left\{1-\sum_{j=1}^{k-i} \frac{\lambda_{i j}^{2}}{n-i}\right\}^{\frac{n-k-2}{2}} \prod_{j=1}^{k-i} \frac{d \lambda_{i j}}{\sqrt{n-i}}\right] .
\end{gathered}
$$

However $\left\{1-\sum_{j=1}^{k-i} \frac{\lambda_{i j}^{2}}{n-i}\right\}^{\frac{n-k-2}{2}}$ converges to $\exp \left[-\frac{1}{2} \sum_{j=1}^{k-i} \lambda_{i j}^{2}\right]$ uniformly in $\lambda_{i j}$ as $n \rightarrow \infty$. Thus, the integral (3.4) including the normalization constant converges to $\exp \left[-\frac{1}{2} \sum_{i, j} t_{i j}^{2}\right]$ as $n \rightarrow \infty$.

Thus, we have proved 
Theorem. The projective limit space $(\Omega, \mathcal{B}, \mu)$ is isomorphic with the Gaussian measure $g$ on $R^{\infty \infty}$. Namely, there exists a measurepreserving one-to-one mapping $\psi$ from a suitable subset $\tilde{\Omega}$ of $\Omega$ onto a suitable subset $\tilde{R}^{\infty \infty}$ of $R^{\infty \infty}$ where $\mu(\tilde{\Omega})=g\left(\tilde{R}^{\infty \infty}\right)=1$, and $\psi$ preserves matrix elements in some sense.

\section{References}

[1] Yamasaki, Y., Invariant measure of the infinite dimensional rotation group, Publ. RIMS Kyoto Univ. 8 (1972/73), 131-140 (this issue).

[2] Hida, T. and H. Nomoto, Gaussian measure on the projective limit space of spheres, Proc. Japan Acad. 40 (1964), 301-304.

[3] Umemura, Y. and N. Kôno, Infinite dimensional Laplacian and spherical harmonics, Publ. RIMS Kyoto Univ. 1 (1966), 163-186.

[4] Shale, D., Invariant integration over the infinite dimension orthogonal group and related spaces, Trans. Amer. Math. Soc. 124 (1966), 148-157.

[5] Shimomura, H., Invariant measure on the $\infty$-dimensional orthogonal group, A report submitted to Kyoto University, 1970 (in Japanese). 
